Fecha de recepción: noviembre 2018 Fecha de aceptación: marzo 2019 Versión final: julio 2019

\section{La Internacionalización de la Educación Superior a través de las revistas científicas digitales en América Latina}

\author{
Micaela Persson *
}

Resumen: El objetivo del trabajo es identificar las oportunidades y desafíos que enfrenta la circulación del conocimiento científico para Latinoamérica, a través de las revistas científicas digitales bajo parámetros abiertos (Open Access, OA). El análisis se inicia con el impacto de la tecnología en el desarrollo de la internacionalización de la educación superior. Bajo una investigación exploratoria/descriptiva, se utiliza una metodología cualitativa sobre la base de datos de Latindex y de Web of Science (ex ISI Thomson).

Palabras clave: Publicaciones Electrónicas - Universidades - Conocimiento - América Latina.

[Resúmenes en inglés y portugués en la página 164]

${ }^{(*)}$ Lic. en Relaciones Internacionales (UNICEN). Doctoranda en Educación Superior (Universidad de Palermo). Coordinadora de Investigación, MBA, Universidad de Palermo, Buenos Aires, Argentina. Docente de grado en UCES, posgrado en Universidad de Palermo e investigadora tiempo completo.micapersson@gmail.com

\title{
Introducción
}

El objetivo del trabajo, es identificar las oportunidades y desafíos que enfrenta la circulación del conocimiento científico para Latinoamérica, a través de las revistas científicas digitales bajo parámetros abiertos (Open Access, OA). Dichas revistas constituyen el elemento principal en la comunicación de los resultados de investigación, tanto como para difundir y para posicionar su prestigio a través de las citaciones y el factor de impacto de sus publicaciones. Con el fin de articular la investigación, la misma se divide en diversas secciones, comenzando por la actualidad de la internacionalización de la educación superior en forma general para llegar a la región latinoamericana.

Bajo una investigación exploratoria, se utiliza una metodología de tipo cualitativa sobre la base de datos de Latindex para identificar y analizar la producción científica en Latinoamérica. Recurrir a esta base de datos permite conocer índices en espacios que tradicionalmente no son analizados, ya que la mayoría de los estudios realizados en este campo 
toman como base la información contenida en el Web of Science, donde la gran mayoría de las revistas producidas en América Latina no figuran. También se utilizan fuentes secundarias de trabajos que analizan redes de co-autoría, con el objetivo de indagar sobre la colaboración científica en Latinoamérica. La unidad de análisis es el documento (paper), ya que a partir de firmas (autores) y su procedencia, se puede dar cuenta de la colaboración científica entre países (esto es, los documentos con autores provenientes de al menos dos países diferentes). A su vez, el análisis incluye la base WoS (ex ISI Thomsom), con el fin de comparar información disponible y resultados encontrados con publicaciones en inglés. Seguidamente, son analizados los resultados de la investigación realizada por Laakso y Björk (2012).

\section{La Internacionalización de la Educación Superior}

La universidad es el centro de análisis de diversos abordajes que reflexionan sobre cuál es su rol en la actualidad. Desde la óptica de la economía basada en el conocimiento, se destaca el papel clave de la misma, por su capacidad de generar y democratizar dicho conocimiento.

En este marco, la investigación triangula la teoría de la internacionalización de la educación superior desde los distintos enfoques (contextual, institucional y crítico) con una mirada desde la perspectiva latinoamericana vinculada a la colaboración científica y al OA por medio de un análisis documental basado en revistas científicas regionales y las bases de datos que agrupan a estas.

Es ya un lugar común manifestar que nos dirigimos hacia una economía global. El mundo es cada vez más inter dependiente en lo que respecta al comercio, la cultura y la comunicación. Hay una institución que siempre ha sido global y que, luego de medio milenio, sigue constituyendo una fuerza poderosa en el mundo. Esa institución es la universidad. En la sociedad del siglo XXI, basada en el conocimiento, la universidad seguirá estando en el centro del desarrollo económico y cultural (Altbach, 2009, p. 23).

Entonces, ¿por qué se trabaja bajo el concepto de internacionalización de la educación superior sobre finales del siglo XX? Hacia 1970 se inicia una profunda reestructuración de la economía capitalista, afectando el orden económico, político-social y tecnológico mundial. Entre otros aspectos, los países industrializados comenzaron a vislumbrar en la investigación y el desarrollo de nuevas tecnologías, herramientas claves para superar la crisis y reafirmar su hegemonía a nivel mundial (Persson, 2008).

Para la década del noventa, los avances tecnológicos del cambio de paradigma basado en el conocimiento y la información ${ }^{1}$, se consolidaron como parte cotidiana del trabajo e irrumpieron en las organizaciones, ayudados por la flexibilidad y la adaptabilidad -cruciales para garantizar la velocidad y la eficiencia de la reestructuración- (Piñero, 2003 y Persson, 2018) y las universidades no estuvieron exentas de tales cambios que se profundizan y avanzan constantemente. 
El análisis de este nuevo entorno se denominó internacionalización de la educación superior $^{2}$, definido por los referentes en el tema (Altbach, 2009 y Knight, 2006) como un proceso que no refiere exclusivamente a esa característica cosmopolita intrínseca de la universidad, sino que se vincula con la configuración de un escenario particular: un mercado académico global, con la utilización del idioma inglés como lenguaje internacionalmente aceptado para la comunicación de la investigación y docencia; la educación a distancia y el uso de Internet a partir de las tecnologías digitales.

En palabras de Altbach (2009) estas relaciones se dan en un contexto de desventaja tecnológica, financiera y estructural, que en su interrelación configuran tensiones en el proceso de internacionalización. “... estas publicaciones, escriben en las lenguas en que las principales revistas publican -principalmente en inglés- $y$ hasta cierto punto orientan su investigación hacia los temas que prefieren las mismas" (p. 81). En tanto, se advierte que este fenómeno ha sido analizado principalmente por autores de procedencia canadiense, europea y estadounidense, presentándose una escasez de trabajos de autores latinoamericanos, lo que trajo aparejado que, en la década de los años noventa, la internacionalización de las universidades de la región latinoamericana estuviera signada por la visión de la cooperación internacional promulgada por Estados Unidos o Canadá y por el proceso de Bolonia iniciado en el continente europeo (Oregioni, 2015).

El contexto internacional, conduce el camino de la investigación hacia América Latina, los desafíos y tensiones que se encuentran en un campo atravesado por el desarrollo tecnológico. Abba, López y Taborga (2010) advierten en este sentido, la diferenciación entre la internacionalización física y virtual. En tiempos actuales, conjuntamente con los procesos históricos de 'internacionalización hacia afuera', entendida como la movilidad física de los estudiantes o docentes hacia otros países, cobran centralidad los procesos de internacionalización en casa'. Con este concepto se hace referencia a la 'movilidad ficticia', es decir, a la modalidad por la cual los estudiantes concurren a sedes de universidades extranjeras sin abandonar su país de origen. La 'internacionalización en casa' hace referencia también a la 'movilidad virtual', es decir, a la educación a distancia por correspondencia, teléfono e Internet.

En este marco cobran relevancia las revistas científicas que son evaluadas bajo parámetros de inclusión de investigadores externos y principalmente de universidades extrajeras. Así el conocimiento se externaliza y comparte, sin tener que desplazarse físicamente. Los call for papers en línea y sistemas como el OJS (Open Journal System), permiten una expansión de la información al alcance de un click o un correo electrónico. El camino recorrido en conjunto con la aplicación de la tecnología en la comunicación y la educación, conllevan al proceso de la investigación colaborativa de revistas científicas, permitiendo una mayor circulación y acceso al conocimiento, de la mano de las tecnologías ya desarrolladas (Internet, base de datos, plataformas en línea, etc.), utilizadas en el campo (motores de búsqueda, identificación de autores $-\mathrm{DOI}^{3}-$, factores de impacto, etc.) y buenas prácticas aplicadas (acceso abierto, no comercial). 


\section{Tecnología, acceso abierto y colaboración}

El marco internacional que inicia el análisis, se caracteriza por tres procesos que convergen entre sí: el papel cada vez más importante que asume el conocimiento a nivel global, un mercado de trabajo para personas cada vez más calificadas y el aumento de la interconectividad entre los productores y consumidores de conocimiento dado por el auge de las tecnologías de la información y comunicación; derivándose de lo anterior una nueva geopolítica global de conocimiento (García Guadilla, 2010).

Más allá de la ampliación de las redes gracias a la utilización de las tecnologías digitales, cabe destacar que la colaboración científica permite ser medida bajo herramientas que otorgan los catálogos y directorios que posibilitan cruzar información digitalizada, que marcan una diferencia radical con la era analógica vinculada con los libros o revistas impresas. Estos avances tecnológicos, por tanto, han transformado la forma habitual de producción: los medios tradicionales que podrían ser identificados como "literatura gris", han venido cediendo espacio a la producción en revistas científicas con mayor potencial de difusión y alcance. Al respecto, cabe destacar la reciente comunicación de Latindex donde comunica a los Editores que a partir de 2018 enfocará sus esfuerzos de calificación a las revistas en línea, con base en el sistema de valoración actualizado, para conformar el Catálogo 2.0, suspendiendo hasta nuevo aviso la calificación de revistas impresas.

Cuestiones como estas reorientan las estrategias de trabajo de los científicos, que poco tienen que ver con las prácticas de hace diez o veinte años (Aguado-López, Rogel-Salazar, Garduño-Oropeza, et. al., 2009, p. 3); y de la mano de dichas prácticas también los dispositivos de comunicación a los que recurren los científicos han experimentado radicales cambios. Gran parte de ellos se relaciona con el uso cada vez más extendido de las tecnologías de la información, como ya se señaló, lo que permite afirmar que se asiste a una transición del papel al pixel en lo que a comunicación científica se refiere; así cada día es más extensivo el uso de revistas, sobre todo en formato electrónico, como dispositivo para comunicar resultados de investigación.

La difusión y el prestigio en este nuevo marco, están asociados directamente a la participación en bases de datos comprensivas internacionales y regionales. El alcance es medido bajo el Factor de Impacto, que surge de la base conocida en el ámbito ISI (Institute for Scientific Information) Thomson/Web of Science en donde el grueso de la investigación se centra en un grupo de países, principalmente en Estados Unidos y Europa. Así de las 100.000 revistas científicas en todo el mundo, sólo 3.000 se encuentran indizadas en la base ISI, que registra las publicaciones internacionales "importantes". La mayor parte se edita en lenguas dominantes, fundamentalmente en inglés (Altbach, 2009). En este marco, los académicos se someten a evaluaciones que requieren de publicaciones en revistas indizadas, internacionales y con co-autorías de universidades por fuera de la región y en un idioma diferente, generando barreras a superar en un contexto en donde la producción científica en universidades latinoamericanas y las contribuciones académicas entre pares no se caracterizan por su abundancia.

Hasta la actualidad, los parámetros de evaluación internacional, entendidos como apertura geográfica, realizados por los sistemas latinoamericanos (como Latindex, Núcleo Básico CONICET y Redalyc) analizan la publicación de autores y evaluadores externos 
a la unidad editora y cantidad de miembros del comité editorial residentes fuera del país, sin mención de las contribuciones de otras publicaciones o artículos de revisión, con referencia específica a pares o instituciones procedentes de América Latina. Países como Alemania se están plantando frente a editoriales, como Elsevier con su modelo lucrativo tradicional, apoyando un modelo disruptivo, abierto, democrático y accesible. A nivel latinoamericano, cabe señalar los esfuerzos realizados por Latindex (Sistema Regional de Información en Línea para Revistas Científicas de América Latina, el Caribe, España y Portugal, 2018) en cuanto a mantener la accesibilidad y difusión de los contenidos de las revistas bajo parámetros abiertos (modelo OA) y no comerciales, intentando superar la paradoja del investigador en la "era de la información y el conocimiento": pagar para publicar, publicar para pagar y pagar para leer.

El mencionado modelo de OA, es una forma revolucionaria de proporcionar acceso a la literatura académica de revistas hecha posible por Internet. En el contexto de la publicación académica, es un término ampliamente utilizado para referirse al acceso en línea sin restricciones a artículos publicados en revistas académicas. Hay dos maneras distintas para que los artículos académicos estén disponibles OA: directamente proporcionados por el editor de la revista (Gold Open Access), o indirectamente por ser cargados y puestos a disposición libre y gratuitamente en otro lugar de la Web (Green Open Access) ${ }^{5}$. Ambas opciones aumentan los lectores potenciales de cualquier artículo a más de mil millones de personas con acceso a Internet e indirectamente aceleran la difusión de nuevas ideas de investigación. Si bien la mayoría de las revistas de acceso abierto no les cobran a los autores por los servicios brindados, una creciente minoría de revistas que operan profesionalmente cobran tarifas que van desde 20 a 3800 USD (dólar estadounidense), con un promedio estimado de 900 USD (Laakso y Björk, 2012). Los orígenes de este nuevo modelo no sólo se vinculan con la aparición de Internet, sino también con que el OA está estrechamente relacionado con los desarrollos de empresas de contenido multimedia y su filosofía está bien alineada con el principio de apertura fundamental de la ciencia misma, así como con las ideologías detrás de Wikipedia y el software de código abierto (Laakso y Björk, 2012). De tal manera, la clave está en la colaboración. Miles de plataformas electrónicas de intercambio de productos y servicios se expanden a toda velocidad en un abierto desafío a las empresas tradicionales, sus principales exponentes son Uber, Airbnb, Waze, basadas en modelos de negocio ${ }^{6}$ que se caracterizan por ser dinámicos, es decir, se adaptan a los cambios en el mercado. Otra de las consideraciones de este modelo de negocio es que las plataformas que acercan a los pares no poseen ningún bien ni son las prestadoras directas del servicio. Airbnb no es dueño de las habitaciones que ofrece ni Uber es dueño de los autos que dan el servicio de transporte. Por esto es que el éxito de este modelo de negocio depende en gran medida de la construcción de confianza, así como del valor agregado que otorga la plataforma a la transacción, bien sea ahorro de tiempo, menores costos o las garantías en caso de que algo falle (Pedraza, 2018).

Sin embargo, Laakso y Björk (2012) advierten en su investigación lo que genera que la publicación científica sea distinta es la influencia que tienen el prestigio y el ranking de la revista en la selección de las mismas para los autores que envían manuscritos de artículos. También existen intereses creados para preservar el status quo del mercado actual de suscripción entre las partes interesadas, con los editores dominantes que ven a OA como 
una amenaza potencial para el resultado final. Según el estudio de los mencionados autores, la publicación de revistas de acceso abierto está alterando el modelo dominante de suscripción de publicación científica, habiendo crecido rápidamente en la participación anual relativa de artículos de revistas publicadas durante el año 2000 y el 2011. Lo anterior mencionado, permite plantear ¿por qué en la "era de la democratización de la información”, el OA, la colaboración, las plataformas digitales, aplicaciones y descargas sin costo (free), el correo electrónico, etc., la circulación del conocimiento a nivel internacional se encuentra concentrado y liderado por un grupo de países? Siguiendo la investigación de Laakso y Björk (2012), han aplicado diferentes métodos para hacer frente a la falta de datos cuantitativos fácilmente disponibles para estudiar el fenómeno de OA, que van desde el conteo manual de artículos con uso intensivo de mano de obra, hasta el rastreo web automatizado.

La proporción de OA Gold en 2003 fue del 2,9\% para los artículos incluidos en Thomson Reuters Web of Knowledge. En 2006 (según datos de UlrichsWeb y DOAJ) ${ }^{8}$, la participación de OA Gold creció al 8.1\% y una participación de OA Green de 11.3\% resultó en una participación combinada de OA de 19,4\%. Para los artículos de 2008, se midió que la cuota OA Gold de Thomson Reuters Web of Knowledge era del 6,6\% y la OA Green del 14\%, lo que arrojaba una cifra de OA total del 20,6\%. También para 2008, un estudio a gran escala basado en revistas en inglés enumeradas en el DOAJ calculó que se publicaron 120,000 artículos OA a través de revistas OA inmediatas completas o como artículos híbridos individuales de OA. El primer estudio longitudinal integral sobre el volumen de artículos publicados por revistas OA inmediatas completas en el DOAJ dio como resultado una tasa de crecimiento anual promedio del 30\% entre 2000 y 2009, con unos 191,000 artículos publicados durante 2009. Otro estudio longitudinal, que incluye OA Gold y Green, produjo una participación total de OA del 23,1\% para los artículos indexados de Thomson Reuters Web of Knowledge publicados durante 2010. Fuera de este estudio de 2010 de Thomson Reuters Web of Knowledge, no hay mediciones exhaustivas para el volumen de OA desde 2009. De este modo, es posible observar el amplio crecimiento y sostenimiento de las prácticas de los científicos en publicar sus artículos en revistas de OA.

Para respaldar el análisis de las revistas muestreadas, Laakso y Björk (2012) utilizaron datos adicionales de Scopus y Thomson Reuters Web of Knowledge, además de los datos que se encuentran disponibles a través de DOAJ. Siguiendo el modelo mencionado, el análisis del actual trabajo de investigación, supone que todas las publicaciones de revistas digitales latinoamericanas se encuentran en la base de datos de Latindex. Hay revistas OA no indexadas en esta base de datos, pero identificarlas sistemáticamente no es factible. Debido a que la mayoría de las 8.175 revistas en línea enumeradas en Latindex ${ }^{9}$, no se incluyeron en ningún servicio de indización que pudiera realizar un seguimiento fiable de la publicación de sus artículos, ni el año exacto en el que las revistas basadas en suscripción se convirtieron en OA.

Los índices disponibles en la base de Latindex (2018), se dividen en: tema, región, país, título, editorial e indización. Como se puede advertir, el principal impedimento para observar datos de colaboración científica es que la plataforma no dispone de tal segmentación, por lo que se debe recurrir al trabajo manual (como lo han realizado algunas investigaciones de manera parcial o utilizando el muestreo aleatorio que se toman como referencia para la 
presente investigación). En esta línea, se destaca el trabajo desarrollado por la Universidad Autónoma del Estado de México) cuyas revistas se encuentran transitando hacia formas de trabajo colaborativas, mediante la formación de redes autorales no sólo nacionales sino ya, también, a escala internacional (Aguado-López, et al., 2009, p. 253-254).

Con respecto a la base WoS (ex ISI Thomsom), no es tarea sencilla desglosar la intrincada plataforma Web de la empresa Thomson Reuters, para clasificar dónde mirar un determinado producto y en qué consiste el mismo. Sin embargo, esto es lo que se deduce de la página oficial de la empresa10 y de sus informes anuales, donde se muestran las grandes áreas donde maneja sus productos, a los cuales denomina: "información inteligente" (Información Tecnológica, 2013). Thomson Reuters Web of Knowledge es una plataforma integrada de propiedad de Thomson Reuters que incluye todos los servicios de la empresa relacionados con difusión del conocimiento. En esta plataforma se incluyen varios de los productos de Thomson Reuters tales como: Conference Proceedings Citation Index, Current Contents, Journal Citation Reports, Medline, Scholar-One Products, Science Citation Index, Science Citation Index Expanded, Social Sciences Citation Index y Web of Science, entre tantos otros. Algunos de estos productos están creados para satisfacer a diversos usuarios y por eso que un producto puede formar parte de otro, como ocurre con la WoS que incluye por ejemplo todas las revistas del Science Citation Index Expanded (SCIE), del Social Sciences Citation Index (SCCI) y del Arts \& Humanities Citation Index (AHCI).

La Web of Science corresponde a una base de datos con información ya procesada sobre aspectos del mayor interés en la investigación sobre más de 12 mil revistas y unas 159 mil actas de congresos. La información está clasificada en 256 categorías en todas las áreas de la ciencia, las artes, las ciencias sociales y las humanidades. Trae los enlaces a otras bases de datos de Web of Knowledge y a los enlaces de los artículos originales que incluye en sus análisis (Información Tecnológica, 2013) ${ }^{11}$. Por lo tanto, la Web of Science ${ }^{12}$ es un índice académico de citaciones en línea que provee la empresa Thomson Reuters a través de su plataforma Web of Knowledge. Las revistas que están incluidas en la Web of Science corresponden a las que hace unos años estaban en el listado del ISI, que luego fue expandido a ISI-expanded y que finalmente pasara a integrar las bases de datos y productos de Thomson Reuters ${ }^{13}$.

El análisis de Laakso y Björk (2012), muestra que los primeros años de la publicación de OA fueron en gran parte impulsados por sociedades científicas, asociaciones profesionales, universidades y sus departamentos, así como por científicos independientes. La publicación de artículos estuvo centrada en tres categorías diferentes: revistas en línea que requieren un cargo de procesamiento de artículos, journals en línea que no requieren un cargo por procesamiento de artículos y revistas que aún producen versiones impresas para suscriptores, pero tienen todos los artículos disponibles OA en línea. Es importante señalar que las revistas que aún producen una versión impresa también pueden requerir un cargo por procesamiento de artículos además de tener ingresos de las suscripciones (sin embargo, tal diferenciación no se proporciona aquí debido a la relativa rareza de tales revistas, así como también al objeto de enfocarse específicamente en estos tres modelos comerciales mutuamente excluyentes). 
Gráfico 1. Cantidad de editores por regiones geográficas 2000-2011

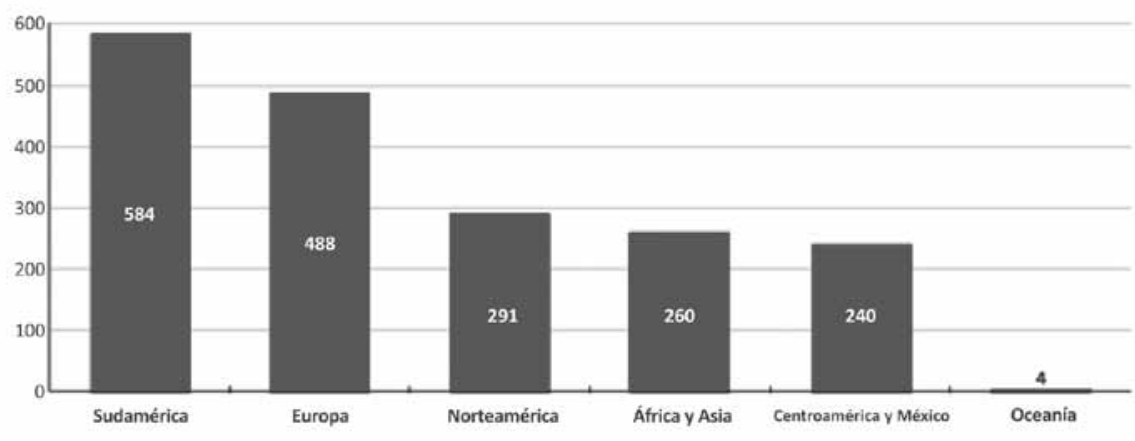

Fuente: Laakso y Björk (2012)

De las revistas en línea incluidas en Latindex (total 8.175) se resalta que el país con mayor cantidad de revistas en línea es España (18\% del total), seguido por los países latinoamericanos de Brasil (33\%), Argentina (11\%), México (10\%), Chile (6\%), continuando por Portugal (3\%), Costa Rica, Cuba y Venezuela (2\%), Perú y Colombia (1\%). Dichos países, superan el piso de las 100 revistas indizadas, el resto de organismos internacionales y países, no alcanzan tal cantidad y representan el $10 \%$ del total.

Gráfico 2: Índice por país de revistas en línea

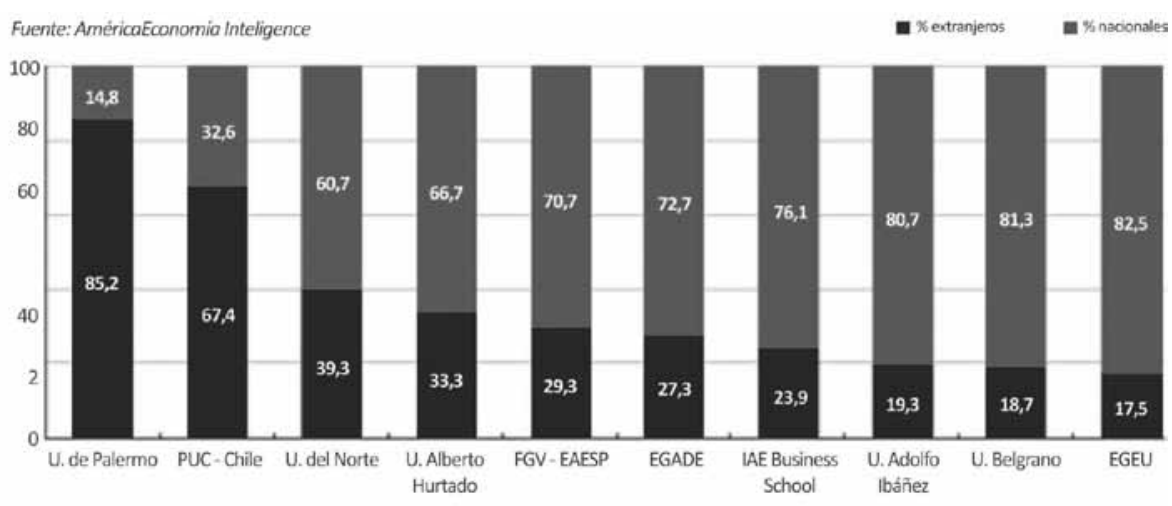

Fuente: Latindex $(2018)^{14}$ 
En suma, como se ha visto desde el año 2008 las publicaciones exclusivas en línea han tenido un fuerte crecimiento, mientras que la producción de OA proporcionada por las revistas que emiten una versión impresa se ha estabilizado a volúmenes anuales de entre 100.000 y 110.000 artículos (Laakso y Björk, 2012). Las revistas impresas incluyen muchas registradas con portales dedicados como SciELO, Redalyc y J-Stage proporcionan la plataforma técnica para la publicación electrónica (sistema OJS). Como sostienen estos autores, casi la mitad de todos los artículos completos de OA publicados durante 2011 estuvieron fuera de Scopus y dos tercios fuera de Thomson Reuters Web of Knowledge, lo que significa que una gran parte de los artículos OA carece de cobertura en los principales índices de publicación.

\section{Conclusiones}

En el transcurso de las últimas dos décadas, la publicación de revistas OA ha crecido de manera universal a través de diversos tipos de editoriales, regiones geográficas y disciplinas científicas. Esto ha resultado en un contexto de avance de nuevas tecnológicas de información y comunicación, que sin embargo, a pesar de todas las dimensiones estudiadas que muestran aumentos en la producción anual de artículos durante los últimos años, los resultados del estudio de Laakso y Björk (2012), muestran que el crecimiento no ha sido uniforme en todos los ámbitos. La publicación de OA parece estar en una fase de crecimiento muy dinámica, con grandes cambios en la composición interna que suceden en un lapso de tiempo relativamente corto y dispar dependiendo de la región editora.

Este primer análisis de bases lleva a arrojar números de manera inductiva. Lo que se puede considerar una debilidad es la dependencia del muestreo en lugar de la cobertura completa de la población, sin embargo, tal enfoque no es factible con las herramientas de indización actualmente disponibles y la recopilación manual de datos para más de 8.000 revistas. A su vez, para futuras investigaciones es necesario considerar los índices de otra base importante para Latinoamérica, como la correspondiente a Redalyc.

Como resaltan los autores anteriormente analizados, ya no parece ser una cuestión de si OA es una alternativa viable al modelo de suscripción tradicional para la publicación académica de revistas; la pregunta es más bien cuándo la publicación OA se convertirá en el modelo principal. En cuanto al aporte desde el lado de Latinoamérica, queda abierto el interrogante de cómo afrontarán las universidades en el proceso de Internacionalización de la Educación Superior su capacidad de ser protagonista en la generación y circulación del conocimiento, con una visión superadora de la dicotomía centro - periferia del sistema internacional dominado por el idioma inglés.

\section{Notas}

1. Estos conceptos abren diversos debates sobre la "sociedad del conocimiento" (Drucker, 1969) y "sociedad de la información”. Castells (1999) advierte al respecto: la difusión y desarrollo de ese sistema tecnológico ha cambiado la base material de nuestras vidas, por 
tanto la vida misma, en todos sus aspectos: en cómo producimos, cómo y en qué trabajamos, cómo y qué consumimos, cómo nos educamos, cómo nos informamos y entretenemos, cómo vendemos, quién manda, quién se enriquece, quién explota, quién sufre y quién se margina. Las nuevas tecnologías de información no determinan lo que pasa en la sociedad, pero cambian tan profundamente las reglas del juego que debemos aprender de nuevo, colectivamente, cuál es nuestra nueva realidad, o sufriremos, individualmente, el control de los pocos (países o personas) que conozcan los códigos de acceso a las fuentes de saber y poder.

2. En ese sentido, Padilla López (2005) argumenta que no puede existir una definición única para representar un fenómeno tan complejo y en constante cambio.

3. DOI (Digital Object Identifier): identificación de material digital, es un código único que tienen algunos artículos extraídos de bases de datos en la web (American Psychologycal Association, 2010).

4. Es el caso de las llamadas revistas "ISI", que por un tiempo fueron revistas "ISI Web of Science" y que actualmente son simplemente revistas "Web of Science" (WoS), de la empresa Thomson Reuters. Probablemente haya que aguardar que los administradores de la investigación en nuestros países cambien en los formularios y en las bases de todos los concursos el término "revistas ISI" por "revistas WoS". En este cambio ha habido sin duda confusión de términos que ha llevado a muchos investigadores y a administradores de la investigación a mezclar conceptos y términos que son diferentes pero que pocos aclaran. Información obtenida de http://dx.doi.org/10.4067/S0718-07642013000500001, el 10 de julio de 2018.

5. Teniendo en cuenta la diferenciación que realizan los autores, para la presente investigación no se tienen en cuenta los casos de Green Open Access.

6. La presente investigación no desarrolla el debate de los nuevos modelos de negocios; su mención se refiere al contexto del uso de las TICs actuales en la "nueva era de colaboración". 7. o que se sabe sobre los primeros años de OA, tanto Gold como Green, es principalmente a través de una serie de estudios independientes que proporcionan un abordaje de años individuales basados en el muestreo de varios índices de publicación. El hecho de que los estudios se hayan basado en la prevalencia de OA en diferentes índices de publicación y en los diversos métodos de muestreo adoptados, hace que las comparaciones o la composición del desarrollo longitudinal sean inexactas.

8. Directory of Open Access Journals (DOAJ) es un directorio en línea curado por la comunidad que indiza y proporciona acceso a revistas de alta calidad, de acceso abierto y revisadas por pares. DOAJ es independiente. Toda la financiación se realiza a través de donaciones, el $40 \%$ de las cuales proviene de patrocinadores y el $60 \%$ de miembros y miembros del editor. Todos los servicios DOAJ son gratuitos, incluidos los indizados en DOAJ (2018).

9. Consultado el 30/09/2018. Disponible en http://www.latindex.org/latindex/inicio

10. Disponible en https://www.thomsonreuters.com/en/products-services.html

11. Dichos números pueden variar, ya que se actualiza regularmente.

12. Disponible en https://clarivate.com/products/web-of-science/

13. Por lo tanto, se debe empezar a nombrar las revistas más apetecidas por los investigadores y las instituciones dedicadas a administrar la investigación en nuestros países 
como revistas WoS. En áreas específicas, sin embargo, se puede seguir hablando de Science Citation Index Expanded (SCIE), del Social Sciences Citation Index (SCCI), o del Arts \& Humanities Citation Index (AHCI). Todos estos sin embargo están incluidos en el WoS y por eso se simplifica la terminología al indicar cuáles revistas deben pertenecer a estos importantes índices. Consultado el 10 de julio de 2018. Basta con decir WoS. Disponible en http://dx.doi.org/10.4067/S0718-07642013000500001

14. Consultado el 30 de septiembre de 2018. Disponible en http://www.latindex.org/latindex/InicioEre

\section{Referencias bibliográficas}

Abba, J.; López, M. P. y Taborga, A. M. (2010). Internacionalización de la Educación Superior: Hacia un enfoque contextualizado, multidimensional y operativo. VI Jornadas de Sociología de la UNLP. Memoria Académica, 1, 1-19. La Plata: UNLP, FAHCE. Recuperado de http://www.memoria.fahce.unlp.edu.ar/trab_eventos/ev.5624/ev.5624.pdf

Aguado-López, E.; Rogel-Salazar, R.; Garduño-Oropeza, G.; Becerril-García, A.; ZúñigaRoca, M. F., \& Velázquez-Álvarez, A. (2009). Patrones de colaboración científica a partir de redes de coautoría. Convergencia. Revista de Ciencias Sociales, 16, 225-258. Recuperado de http://www.redalyc.org/articulo.oa?id=10512244010

Altbach, P. (2009). Educación superior comparada: el conocimiento, la universidad y el desarrollo. Buenos Aires: Universidad de Palermo.

Altbach, P.; Gumport, P. \& Berdahl, R. (2011). American Higher Education in the TwentyFirst Century: Social, Political, and Economic Challenges. Baltimore: The Johns Hopkins University Press.

Altbach, P. (1991). Trends in Comparative Education, Comparative Education Review, 35, 491-507.

América Economía (2017). Ranking de las Mejores Escuelas de Negocios de América Latina. Especial Ranking MBA. 20, 53-63.

American Psychologycal Association. (2010). Manual de Publicaciones de la American Psychological Association (6 ed.). México, D.F.: Editorial El Manual Moderno.

Ander-Egg, E. (1995). Técnicas de Investigación Social (23 ed.). Lumen: Buenos Aires.

Araya, J. (2015). Aportes para los estudios sobre Internacionalización de la Educación Superior en América del Sur. Tandil: Universidad Nacional del Centro de la Provincia de Buenos Aires.

Arnove, R. y Escotet. M.A. (2013). Reflexiones y tendencias de la educación comparada e internacional. En AA.VV. (ed.) Conversaciones con un maestro (Liber Amicorum). Madrid: Ediciones Balán, Jorge (Ed) (2014) América Latina y su nueva Economía del Conocimiento. Buenos Aires: Universidad de Palermo y Cátedra UNESCO-UNU.

Becher, T. (2009). Tribus y territorios académicos: la indagación intelectual y las culturas de las disciplinas. Barcelona: Gedisa.

Becker, H. (2011). Trucos del oficio: cómo conducir su investigación en Ciencias Sociales. (1 ra. ed.). (1ra. reimp). Buenos Aires: Siglo XXI Editores.

Castells, M. (1996). La era de la información. Economía, sociedad y cultura. México: Siglo XXI. 
Castells, M. (1999). A Era da Informaçao: Economía, Sociedades e Cultura. A Sociedade em Rede, 1 .

Clark, B. (1997). Las universidades modernas: espacios de investigación y docencia. México: Miguel Ángel Porrua.

De Wit, H. (1995). Strategies or Internationalization of Higher Education: A comparative Study of Australian, Canada, Europe and United States. Amsterdam: Asociación Europea para la Educación Internacional.

Directory of Open Access Journals. (2018). Recuperado de https://doaj.org/

Drucker, P. (1969). The Age of Discontinuity: Guidelines to Our Changing Society. New York: Harper \& Row.

Durkheim, E. (1992). Historia de la educación y de las dosctrinas pedagógias. La evoluación pedagógica de Francia. Madrid: La Piqueta.

Elias, N. (1990). Compromiso y Distanciamiento. Ensayos de sociología del conocimiento. Barcelona: Ediciones Península.

Escotet, M. A. (2002). Universidad y Devenir. Buenos Aires: Editorial Lugar.

Escotet, M. A. (2018). Apuntes de clase Educación Superior Comparada. Doctorado en Educación Superior. Universidad de Palermo - Cátedra UNESCO, Buenos Aires, Argentina.

Fernandez, N.; Perez, C.; Marquina, N. y Ailello, M. (Eds.) (2018) La Educación superior universitaria argentina: Situación actual en el contexto regional. Buenos Aires: UNTREF.

Ferreyra, M. M. et al. (Eds) (2017). Higher Education in Latin America and the Caribbean. Washington, D.C.: The World Bank.

Gacel Avila, J. (1999). La política de internacionalización: estrategia de cambio institucional. México: Asociación Mexicana para la Educación Internacional.

Gadamer, H. G. (1984). Verdad y Método II. Salamanca: Sígueme.

García Garrido, J. L. y García Ruiz, J. M. (2012) La Educación Comparada en Tiempos de Globalización. Madrid: UNED.

García Guadilla, C. (2010). Educación Superior Comparada: El Protagonismo de la Internacionalización. Caracas: UNESCO- IESALC y CENDES.

García Guadilla, C. (2010). Heterogeneidad y concentración en las dinámicas geopolíticas del conocimiento académico. Reflexiones y preguntas para el caso de América Latina. En M. Mollis (Ed.). Políticas de posgrado y conocimiento público en América Latina y el Caribe: desafíos y perspectivas. (pp. 135-164). Buenos Aires: Consejo Latinoamericano de Ciencias Sociales.

Gilpin, R. (1990). La Economía Política en las Relaciones Internacionales. Buenos Aires: Grupo Editor Latinoamericano.

Knight, J. (2006). Internationalization: concepts, complexities and Challenges. En J. Forest y P. Altbach (Eds.). International Handbook of Higher Education. Dordrecht: Springer Academic Publishers.

Laakso, M. y Björk, B. (2012). Anatomy of open access publishing: a study of longitudinal development and internal structure. BMC Medicine, 10, 2-9. Recuperado de: doi:10.1186/1741-7015-10-124

Marradi, A.; Archenti, N., Piovani, J. (2007). Metodología de las ciencias sociales. (1ra. ed.). Buenos Aires: Emecé Editores. 
Narváez, J. L. (2018). Clase de Gestión Administrativa y Financiera de la Educación Superior. Doctorado en Educación Superior. Universidad de Palermo, Buenos Aires, Argentina.

Oregioni, M. (2014). Dinámica de la Internacionalización de la Universidad Nacional de La Plata (2005-2012) (Tesis doctoral inédita). Universidad Nacional de Quilmes, Argentina.

Oregioni, M. (2015). La Internacionalización de las Universidades latinoamericanas como objeto de estudio. En J. Araya y M. Oregioni (Comp.). Internacionalización de la universidad en el marco de la integración regional. Tandil: Grafikart.

Padilla López, J. (2005). La Internacionalización como política de cambio y renovación institucional. En: J. Pugliese (Comp.).Educación superior ¿Bien público o bien de mercado? Buenos Aires: MECyT - Secretaría de Políticas Universitarias.

Pedraza, D. (2018). Investigación y propuesta de un plan de negocio para una empresa de servicios -de economía colaborativa- para dueños de mascotas en Bogotá, Colombia (Tesis de maestría inédita). Universidad de Palermo, Buenos Aires, Argentina.

Persson, M. (2008). Drenaje de Cerebros en la Periferia. Causas y Consecuencias en la Globalización y la Revolución Científico - Tecnológica. Estudio del Caso argentino (Tesis de grado inédita). Universidad Nacional del Centro de la Provincia de Buenos Aires, Tandil, Argentina.

Persson, M. (2018). Drenaje de Cerebros y Revoluación Tecnológica. Estudio de caso argntino. Madrid: Editorial Académica Española.

Piñero, F. J. (2003). A inserção da Argentina e do Brasil na Sociedade da Informação Global. Proposta de indicadores de acompanhamento. Tese de doutorado em Sociologia. Faculdade de Cièncias e Letras, Campus de Araraquara, Universidade Estadual Paulista Júlio Mesquita Filho, Brasil.

Quacquarelli Symonds (2017). QS World University Rankings 2017. Top Universities. Recuperado de: https://www.topuniversities.com/universities/universidad-de-palermo\#wurs

Red de Revistas Científicas de América Latina y el Caribe, España y Portugal: www.redalyc. org

Revista de Información Tecnológica. (2013). Editorial. 24, 1. Recuperado de http://dx.doi. org/10.4067/S0718-07642013000500001

Russell, R. (2010). El Estado nación y los actores gubernamentales no centrales: una relacion complementaria. En L. Maira (Ed.), La política Internacional Subnacional en América Latina. Buenos Aires: Libros del Zorzal.

Samaja, J. (2004). Epistemología y Metodología. Elementos para una teoría de la investigación científica. Buenos Aires: Editorial Universitaria de Buenos Aires.

Sistema Regional de Información en Línea para Revistas Científicas de América Latina, el Caribe, España y Portugal: http://www.latindex.org

Theiler, J. (2005). Internacionalización de la educación Superior en Argentina. En H. De Wit, y otros: Educación Superior en América Latina. La dimensión internacional. Banco Mundial.

Thomson Reuters. (2018). Recuperado de https://www.thomsonreuters.com/en/productsservices.html

Viltard, L. (2016). Hacia la Universidad Corporativa (UC). La Configuración del Mercado de Educación Superior y Capacitación Corporativa (CC). Palermo Business Review, 13, 13-32. Web Of Sience. (2018). Recuperado de https://clarivate.com/products/web-of-science/ 
Zárate, R. (1998). Las universidades modernas: espacios de investigación y docencia, de R. Clark Burton. Sociológica. Año 13, 36.331- 337.

\begin{abstract}
The goal of this research is based on identifying the opportunities and challenges facing scientific collaboration for Latin America, through digital scientific journals under open parameters (Open Access, OA). The analysis begins with the impact of technology in the development of the Internationalization of Higher Education. Under an exploratory and descriptive research, a qualitative methodology is used, based on Latindex data and the Web of Science database (ex ISI Thomson).
\end{abstract}

Keywords: Electronic Journals - Universities - Knowledge - Latin America.

Resumo: O objetivo do trabalho é identificar as oportunidades e desafios enfrentados pela circulação do conhecimento científico para a América Latina, através de periódicos científicos digitais sob parâmetros abertos (Open Access, OA). A análise começa com o impacto da tecnologia no desenvolvimento da internacionalização do ensino superior. Na pesquisa exploratória / descritiva, uma metodologia qualitativa é utilizada com base em dados do Latindex e Web of Science (ex ISI Thomson).

Palavras chave: Publicações Eletrônicas - Universidades - Conhecimento - América Latina. [Las traducciones de los abstracts fueron supervisadas por el autor de cada artículo] 